\title{
Extensive spectroscopic and photometric study of HD 25558, a long orbital-period binary with two SPB components
}

\author{
Á. Sódor ${ }^{1,2}$, P. De Cat ${ }^{1}$, D. J. Wright ${ }^{1,3}$, C. Neiner ${ }^{4}$, M. Briquet ${ }^{5}$,
} R. J. Dukes ${ }^{6}$, F. C. Fekel $^{7}$, G. W. Henry ${ }^{7}$, M. H. Williamson ${ }^{7}$, M. W. Muterspaugh ${ }^{7}$, E. Brunsden ${ }^{8}$, K. R. Pollard ${ }^{8}$, P. L. Cottrell ${ }^{8}$, F. Maisonneuve ${ }^{8}$, P. M. Kilmartin ${ }^{8}$, J. M. Matthews ${ }^{9}$, T. Kallinger ${ }^{10}$, P. G. Beck ${ }^{11}$, E. Kambe ${ }^{12}$, C. A. Engelbrecht ${ }^{13}$, R. J. Czanik ${ }^{14}$, S. Yang ${ }^{15}$, O. Hashimoto ${ }^{16}$, S. Honda ${ }^{16,17}$, J.-N. Fu ${ }^{18}$, B. Castanheira ${ }^{19}$, H. Lehmann ${ }^{20}$, N. Behara ${ }^{21}$, H. Van Winckel ${ }^{11}$, S. Scaringi ${ }^{11}$, J. Menu ${ }^{11}$, A. Lobel $^{1}$, P. Lampens ${ }^{1}$, and P. Mathias ${ }^{22}$

${ }^{1}$ Royal Observatory of Belgium, Brussel, Belgium

${ }^{2}$ Konkoly Observatory, Hungarian Academy of Sciences, Budapest, Hungary

${ }^{3}$ School of Physics, University of New South Wales, Sydney, Australia

${ }^{4}$ LESIA, CNRS, Observatoire de Paris, UMPC, Université Paris Diderot, Meudon, France

${ }^{5}$ Institut d'Astrophysique et de Géophysique, Université de Liège, Liège, Belgium

${ }^{6}$ Department of Physics and Astronomy, The College of Charleston, Charleston, SC, USA

${ }^{7}$ Center of Excellence in Information Systems, Tennessee State University, Nashville, TN, USA

${ }^{8}$ Department of Physics and Astronomy, University of Canterbury, Christchurch, New Zealand

${ }^{9}$ Dept. of Physics and Astronomy, University of British Columbia, Vancouver, BC, Canada

${ }^{10}$ Institute for Astronomy, University of Vienna, Vienna, Austria

${ }^{11}$ Instituut voor Sterrenkunde, K. U. Leuven, Leuven, Belgium

${ }^{12}$ Okayama Astrophysical Observatory, National Astronomical Observatory, Okayama, Japan

${ }^{13}$ Department of Physics, University of Johannesburg, Johannesburg, South Africa

${ }^{14}$ Dept. of Phys., Potchefstroom Campus, North-West University, Potchefstroom, South Africa

${ }^{15}$ Department of Physics and Astronomy, University of Victoria, Victoria, BC, Canada

${ }^{16}$ Gunma Astronomical Observatory, Takayama-mura, Agatsuma, Gunma, Japan

${ }^{17}$ Kwasan Observatory, Kyoto University, Kyoto, Japan

${ }^{18}$ Department of Astronomy, Beijing Normal University, Beijing, China

${ }^{19}$ Department of Astronomy, The University of Texas at Austin, Austin, TX, USA

${ }^{20}$ Thüringer Landessternwarte Tautenburg, Tautenburg, Germany

${ }^{21}$ Université Libre de Bruxelles, Bruxelles, Belgium

${ }^{22}$ Université de Toulouse, UPS-OMP, IRAP, CNRS, Toulouse, France

Abstract. We carried out an extensive photometric and spectroscopic investigation of the SPB binary, HD 25558 (see Fig. 1 for the time and geographic distribution of the observations). The $\sim 2000$ spectra obtained at 13 observatories during 5 observing seasons, the ground-based multi-colour light curves and the photometric data from the MOST satellite revealed that this object is a double-lined spectroscopic binary with a very long orbital period of about 9 years. We determined the physical parameters of the components, and have found that both lie within the SPB instability strip. Accordingly, both components show line-profile variations consistent with stellar pulsations. Altogether, 11 independent frequencies and one harmonic frequency were identified in the data. The observational data do not allow the inference of a reliable orbital solution, thus, disentangling cannot be performed on the spectra. Since the lines of the two components are never completely separated, the analysis is very complicated. Nevertheless, pixel-by-pixel variability analysis of the cross-correlated line profiles was successful, and we were able to attribute all the frequencies to the primary or secondary component. Spectroscopic and photometric mode-identification was also performed for several of these frequencies of both binary components. The spectroscopic mode-identification results suggest that the inclination 
and rotation of the two components are rather different. While the primary is a slow rotator with $\sim 6 \mathrm{~d}$ rotation period, seen at $\sim 60^{\circ}$ inclination, the secondary rotates fast with $\sim 1.2 \mathrm{~d}$ rotation period, and is seen at $\sim 20^{\circ}$ inclination. Our spectropolarimetric measurements revealed that the secondary component has a magnetic field with at least a few hundred Gauss strength, while no magnetic field was detected in the primary.

The detailed analysis and results of this study will be published elsewhere.

Keywords. line: profiles, stars: binaries: general, stars: oscillations (including pulsations), stars: rotation, stars: variables: other

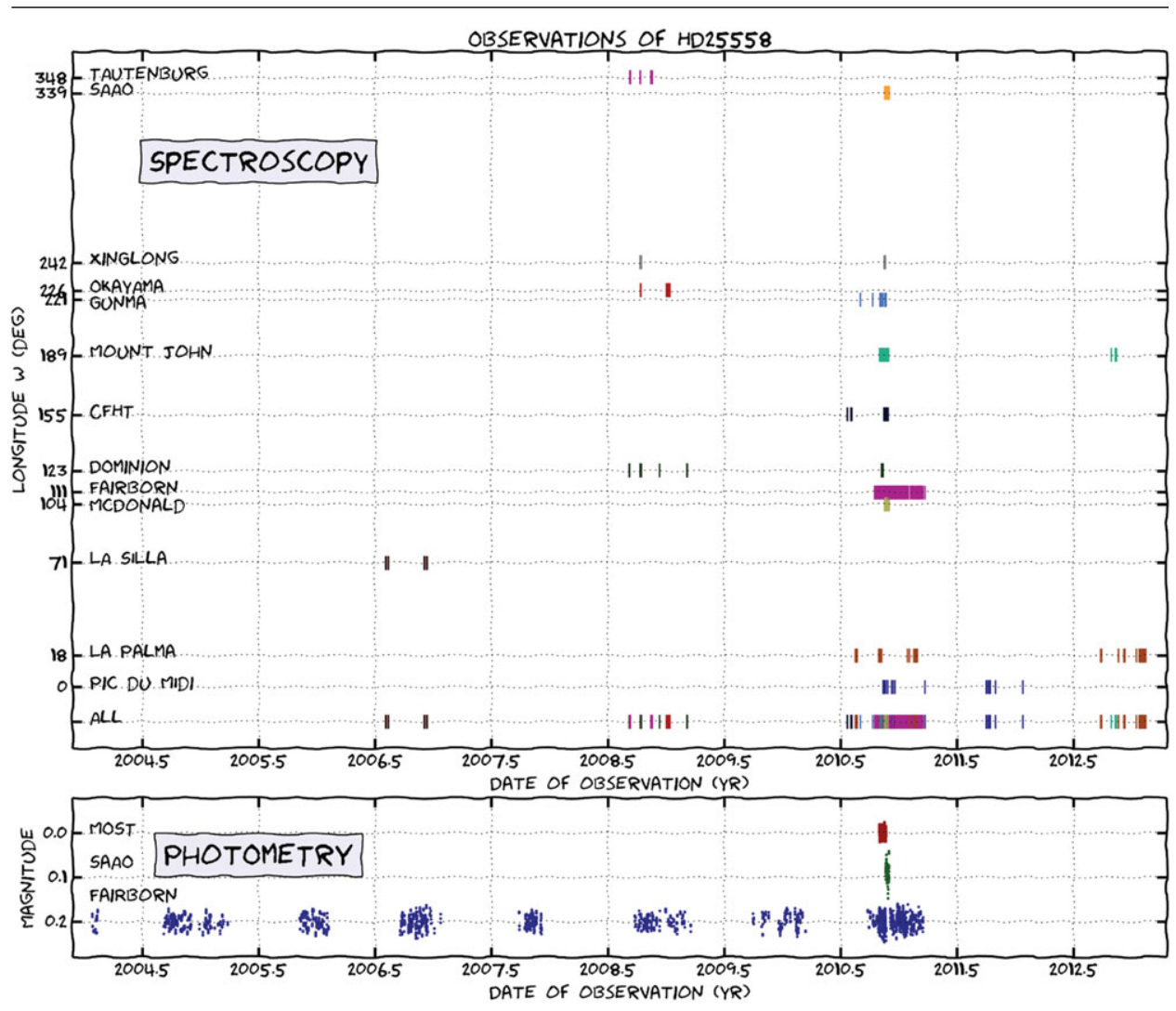

Figure 1. Time and geographic distribution of the spectroscopic and photometric observations of HD 25558. The main campaign was organised in the 2010/11 season.

Acknowledgements. Á.S. acknowledges support of the Belgian Federal Science Policy (project M0/33/029) and of the Eötvös Scholarship from the Hungarian Scholarship Board Office. M.B. is F.R.S.-FNRS Postdoctoral Researcher, Belgium. T.K. acknowledges financial support from the Austrian Science Fund (FWF P23608). The observational data we used in this study were obtained with the following telescopes and instruments: 2-m AST (T13) and 75-cm APT (T5) at Fairborn Observatory, McLellan tel.+HERCULES at Mt John Observatory, Otto Struve tel. at McDonald Observatory, 1.9-m tel.+GIRAFFE and 50-cm tel. at SAAO, Mercator tel.+HERMES at La Palma, 2.2-m tel. at Xinglong Observatory, 1.5-m tel.+GAOES at GAO, Alfred Jensch tel. at Karl Schwarzschild Observatory, 1.9-m tel.+HIDES at OAO, 1.2-m tel.+McKellar at DAO, Bernard Lyot tel.+NARVAL at Obs. Pic du Midi, CFHT+ESPaDOnS at Hawaii, Euler tel.+CORALIE at La Silla, the Canadian MOST satellite. 